\title{
Co-designing Patient-centered Health Communication Tools for Cancer Care
}

\author{
Michael J. Gonzales and Laurel D. Riek \\ Dept. of Computer Science and Engineering \\ University of Notre Dame \\ Notre Dame, IN. 46556 \\ \{mgonza14, lriek\}@nd.edu
}

\begin{abstract}
When oncologists meet their newly diagnosed cancer patients for the first time, they discuss five key topics: diagnosis, prognosis, metastasis, and treatment options and their associated side effects. Studies have shown, however, that while oncologists may feel they have met the informational needs of patients, patients sometimes have different interpretations of what was actually covered. This leads to complications in patient understanding, which are shown to have an impact on patient self-efficacy, confidence, and treatment selection. Due to these major communication issues, particularly during such a sensitive time for patients, we are working in concert with patients and oncologists to design patient-centered health communication tools to help mitigate such problems in clinical communication. Through our discussions with both oncologists and current patients undergoing treatment, we were able to determine key design considerations to help address these issues in clinical communication and shared decision making. In addition, we investigate ways to create personalized health information takeaways for patients, so that they may be better able to process this information after their diagnosis shock has worn off.
\end{abstract}

\section{INTRODUCTION}

An estimated 1.6 million men and women will be diagnosed with some type of cancer in the United States in 2012, with an estimated $41.24 \%$ of all people born today diagnosed with some type of cancer in their lifetime [1]. Patients newly diagnosed with cancer have to absorb a significant amount of information from their oncologists so that they can understand the nature of their disease and appropriately select their treatment plan. It is the oncologist's objective to ensure that five key topics are addressed in a patient's first meeting, including: diagnosis, prognosis (likely outcome), metastasis (likelihood for the disease to spread), as well as treatment options and side effects.

Due to the emotional nature of the discussion, it may be difficult for the patient to process all of the information they are receiving about their disease. Studies show that there is a disconnect between what content patients thought was covered, and what content oncologists thought was covered following these meetings [2]-[5].

Furthermore, interactions with oncologists have significant influence on the outcomes of patient treatment. Positive interactions are shown to have an effect on the approach and decision making areas of treatment, as well as the exchange of trust between both patients and their oncologists [4]. Negative interactions, in contrast, can affect patient self-efficacy and confidence, having a detrimental effect on a patient's approach to treatment [3], [4]. It is important that in these meetings oncologists meet a patient's informational and emotional needs.

When meeting with oncologists for the first time, newly diagnosed patients have a fairly high cognitive load. They need to understand and learn new terminology, conceptualize their treatment options, attempt to visualize many of the concepts discussed during their visit. These tasks are difficult in general, let alone for people emotionally distressed about their diagnosis.

Oncologists also face challenges in these discussions, because a number of factors can contribute to a patient's informational needs during the visit. These include: the patient's cancer type, level of educational attainment, cultural background, emotional state, and age [5]. Furthermore, oncologists need to adjust communication if the patient's treatment will be curative or palliative in nature [6].

Another major challenge is what patients take away with them after these stressful visits. Currently, the take-away information is relatively impersonal, usually in the form of a generic pamphlet [7]. Oncologists often encourage patients to bring tape recorders, or companions to the visits, but in case there is a disagreement in interpretation, these may not always be an effective means to improving what the patient understands from the meeting [2].

Given these challenges, we are designing patient-centered health communication tools (PCHCTs) that will help oncologists and newly diagnosed cancer patients have a more fruitful discussion, and also allow patients to have a personalized takeway. We are working closely with a local oncology clinic and cancer support group, employing participatory design techniques where all major stakeholders are design partners.

In this paper, we present our findings from interviews and design workshops we have conducted with patients with gynecological cancer and oncologists. We focus on addressing the following research questions:

- What are the informational needs of newly diagnosed patients in their first meetings with oncologists?

- Can we meet patient information needs using PCHCTs in the clinical setting?

- What are the needs of the oncologist in the implementation of such interfaces?

- How can we supplement take-home information through use of these interfaces? 


\section{BACKGROUND}

\section{A. Cancer communication}

Given the emotional nature of cancer discussions, it can be difficult for patients to fully understand many of the complex terminologies and concepts relating to their diagnosis. A study analyzing the interpretations between oncologists, newly diagnosed patients, and their companions in triadic clinical interactions show disagreement over the five key topical areas (i.e., diagnosis, prognosis, etc.). The study, conducted by Eggly et al. [2], asked participants to measure their own understanding of what was discussed, as well as the understanding of the other participants in their meeting. Their results indicate that while agreement existed over whether topics were actually covered, disagreement existed among the content of the key topical areas. While each participant tended to show a higher correlation in interpretation with respect to the diagnosis, other topical areas such as treatment side effects showed much lower correlation, indicating potential communication issues [2].

Studies regarding the unmet informational needs of patients and patient satisfaction analyzed the difficulty in conveying such information in a manner that is level with the psychological state of the individual [3], [5]. Patients in some cases may seek out less information relating to their prognosis (likely outcomes) and severity due the highly emotional nature of the content. This, in turn, affects the manner in which the oncologist discusses or presents information. Similar work by Jenkins et al. [8] found that the manner in which individuals categorize good and bad news varies between individuals, with tolerance of bad news also being a dynamic factor.

Despite such differences in patient tolerance, however, Hack et al. [5] found that patients continued to report unmet informational needs, especially with respect to the prognosis, treatment information, and resulting side effects from treatment. Furthermore, Schaefbauer et al. [9] performed an analysis of patient satisfaction in these meetings, which showed an effect of oncologist attentiveness and empathic expression on patient self-efficacy and confidence. The effect of oncologist actions and conveying of information in manners that suit patient needs could lead to better overall patient understanding, satisfaction, and treatment response. As such, design considerations must be taken into account in the deployment of such technology in clinical settings [9].

\section{B. Technology in physician-patient communication}

Breen et al. [10] assessed the effective use of technology in clinical environments for optimal patient-centric care. Findings suggest that clinical and administrative decision support systems should be designed and assessed for improving patient-provider communication [10]. Breen advises that while designing these systems, however, that overall patient engagement and participation be considered in response to increased computer use in clinical settings.

Chen et al. [11] suggests that devices designed to facilitate patient engagement, such as movable computers in the exam room, allows for patients to take a much more active role in their interactions with clinicians. Similarly, Alsos et al. [12] suggest that in clinical environments, secondary users, such as those that may not actually be interacting with a system but are still affected by it, should be considered in the design of such devices or tools. Alsos et al. suggests it is important to consider the secondary user's informational needs by providing adequate feedback, language representation, and non-verbal communication support, similar to work done by Ni et al. [13].

Addressing secondary user needs is major consideration when designing new technologies for older individuals. Piper et al. [14] explored the capability and potential for accessible surface computing in clinical environments for older adults. Similarly in cancer settings, older individuals might prefer written information instead of seeking it on the web [15], however, this does not mean the information they receive should be any less than technology-interested users.

\section{Technology in cancer informational needs}

In addition to technology use in physician and patient communication, patients must do a lot of information seeking beyond what is provided by their oncologist. While an oncologist may provide informational pamphlets and refer the patient to trusted health sources, the patient themselves may need to or choose to do a lot of information gathering on their own. Klasnja et al. [16]-[18] investigated the "unanchored" informational work performed by breast cancer patients in qualitative field studies focused on using mobile technologies to support patient information management of treatment plans and management of questions. Similar work was conducted by Littlechild et al. [19], who assessed women with breast cancer and their information seeking, with $63 \%$ of women choosing websites of their own initiative. Their results also showed $31 \%$ of patients experiencing problems using the internet, illustrating some of the complications associated with information gathering.

Another factor in technology design is the overall physical space of the clinical environment. Unruh et al. [20], [21] assessed the affective nature of the clinical environment itself, and provided key considerations that must be taken into account when designing new information and decisionmaking tools in the exam room. These included limitations for patients in viewing reference materials, bursty communication issues, and challenges in patient preparation for meetings. The work also highlights challenges faced by patients post-visit, including fragmented information, decontextualized notes, and clarificaction problems. The authors suggest the exploration of applications to facilitate agenda management, interactive interfaces for collaboration, and the use of informational space for clarifying information at home.

\section{Designing for patients and oncologists}

Designing for multiple users involves a process of facilitation among designers such that various stakeholder considerations are taken into account [22]. As a result, tools co-designed for various users must take into account each stakeholder's concerns such that their needs are adequately met. Participatory design (PD) is widely used as a design method in applications of technology where stakeholders themselves play an active role in the design process [23]-[27].

PD is an important part of designing for the healthcare environment, as it allows the designer to adequately address stakeholder needs such that both the users' and the designer's 
conceptual models co-align. Its use in design for medical applications can be seen across a number of applications, including the development of tools for cancer patients [17], [18], [20], [21], [28]-[30], children [31], and older adults [23][25], [28], [32].

\section{Methodology}

In order to define requirements for shared-decision making and information tools in diagnosis meetings, we began by working with a specific group of cancer patients. Our participants represent a subset of a gynecological cancer support group in the local area and oncologists from a local cancer clinic to identify the concerns and affective needs for first time meetings of each respective party.

All the following research was approved by the University of Notre Dame Institutional Review Board. All participants gave informed consent to participate, all participant data was appropriately anonymized, and all data was stored securely.

\section{A. Stakeholder recruitment}

1) Oncologists: Our original intention was to perform design workshops with multiple oncologists together; however, we found this was unfeasible given the time requirements for workshops and the busy schedules of the clinicians. Instead we coordinated with three oncologists to perform one-on-one design discussions at the local oncology facility.

In these interviews, we performed contextual inquiries to identify the key concerns oncologists have during their initial meetings with patients, as well as to brainstorm ideas for making it easier for oncologists to relay personalized health information. We interviewed two medical oncologists and one radiation oncologist for variation of expertise and modalities of treatment. Medical oncologists work with patients from the point of diagnosis through the course of treatment, overseeing the patient's treatment in the case of curative care, or guiding them through end-of-life decision making in palliative care. Radiation oncologists focus primarily on the treatment and management of cancers using radiation therapy, in addition to other treatments as prescribed by medical and specialty oncologists.

Oncology participants ranged between 39 and 53 years of age ( $m=48$ years old), were of mixed heritage, and had board certifications including hematology, oncology, internal medicine, and radiation oncology, respectively. Oncology treatment specializations included: chemotherapy, biopsy, hormonal therapies, and gamma knife radiosurgery among others. Participants practised in their field for at least 13 years. Oncology participants were offered compensation, but declined.

The discussions and contextual inquiries with the oncologists were limited to an hour due to their schedules. Our main objective in working with oncologists is to explore ways in which patients may be better informed during and after these initial meetings without causing a major disruptive effect on the oncologists' existing workflow. Furthermore, we do not want to perturb oncologists' existing strategies for organizing these discussions.

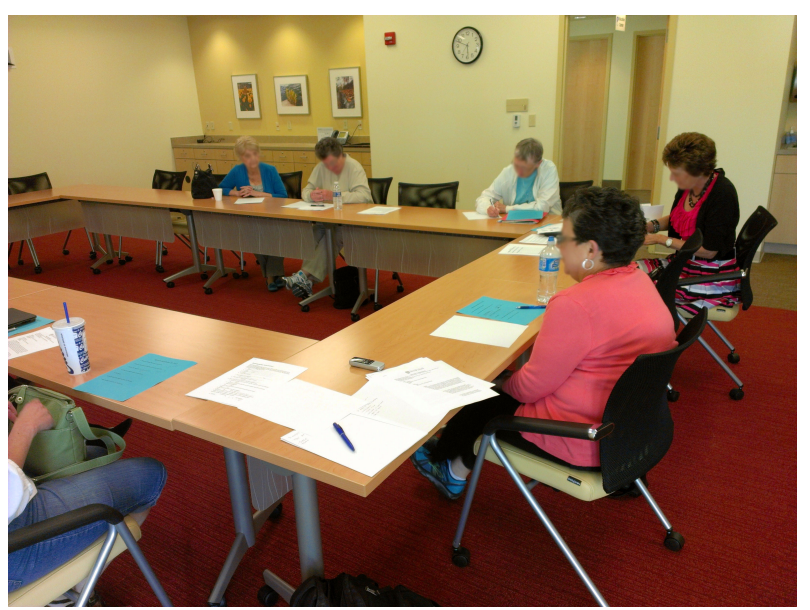

Fig. 1. Design workshop with patients with gynecological cancer.

2) Patients with cancer: We recruited a sample of eight female participants who are either actively undergoing treatment for stage $2 \mathrm{~B}$ to stage $3 \mathrm{C}$ ovarian cancer, or have conquered the disease as a result of treatment. Participants were recruited through local cancer support groups, using paper flyers and word-of-mouth. The group was targeted due to their being one of the most active cancer support groups in our community.

At the time of our study, participants were still actively undergoing treatment were having regularly scheduled followup visits with their oncologists. Participants were between 55 and 75 years of age ( $m=63$ years old), were predominately white or of mixed heritage, and were highly diverse in terms of socioeconomic status and educational attainment. All participants had at least a high school education, with three having received a Bachelor's degree, and one with a Master's degree.

Participants had varying levels of experience with technology and internet use, assessed using a web-literacy instrument by Hargittai et al. [33]. Two participants had little to no experience with computers and the web, one expressed some understanding, while five expressed fluency with various webbased and application topics, such as file extension association.

We conducted a total of three different design workshops, two located on-campus at the University of Notre Dame, and the third following a support group meeting at a local clinic, shown in Figure 1. The maximum turnout at any one of our workshops was seven, and our minimum turnout rate was two participants during our second workshop. Each patient participant was compensated $\$ 15$ for participating in one of our workshops over the course of the study.

\section{B. Patient Design Workshops}

The duration of each workshop was limited to two hours maximum, such as not to overburden patient participants, while still giving them time to be involved in the design process, provide design considerations, and to voice concerns. Much more emphasis was placed on the design process during the workshops with patients to provide better patient-centric informational tools. 


\section{PROCEDURE}

Audio was recorded in all design sessions, which was later transcribed, then analyzed using content analysis techniques involving two independent reviewers to identify major recurrent themes among our audio data. Using the results of our analysis, we identified concerns among participants to define design guidelines important to each stakeholder. Inter-rater reliability is calculated between reviewers to ensure agreement on theme occurence to guide the design of future tools in the clinical setting.

Sessions with oncologists focused on suggestions and concerns in potential tool usage, as well as contextual inquiries in the exam room. Workshops were formatted to focus on four key discussion/collaborative themes, including: past experiences, identifying in-meeting informational needs, design sketching, as well as take-home informational needs.

\section{A. Oncologist discussions}

1) Design Interviews: In these interviews, oncologist partcipants gave an overview of the approach used in their meetings, including preparation and adjustments in communication strategies made depending on the emotional state of the individual they are working with. Oncologists were asked to give design considerations for how PCHCTs could be created to benefit their preparation for these meetings, as well as ideas they would like to see implemented to better inform or educate patients either during or following the meeting. Our goal was to identify the oncologists' main objectives in their discussions with patients, understand the discussion context among various cases, such as palliative and curative treatment plans, as well as to define the requirements oncologists themselves would need in using information tools in the clinical communication setting.

2) Contextual inquiries: We performed contextual inquiries with two medical oncology participants involving the oncologists performing talk-aloud techniques in relation to their actions around the exam room. Our inquiries also involved discussing with oncologists how they might use such a device using a low-tech tablet-like conceptual model in the clinical setting. This provided insight for not only understanding the discussion flow in the exam-room, but also for understanding technological feasibilities for implementation to avoid disruption to the oncologists' workflow.

\section{B. Patient design workshops}

1) Discussion of past experiences: We conducted discussions of participants' experiences in the clinical environment to determine potential problem areas that should be mitigated to enable new patients to have their informational needs adequately met. Care was taken to focus on the unmet informational needs of these meetings to avoid any unwanted burden on the part of our participants. This phase enabled us to identify areas where either a lack of information occurred during the first primary meeting, areas where participants felt overburdened with information, as well as where participants showed disinterest in learning additional information based on personal preference. We also discussed take-home information provided to participants following their initial meeting with their oncologists, as well as the presence and use of technology



I don't know how many people [sit] there with the..deer in headlights look, but I certainly did. I think I heard very little... I was just totally gone. --"Michelle", Age 62, ovarian cancer
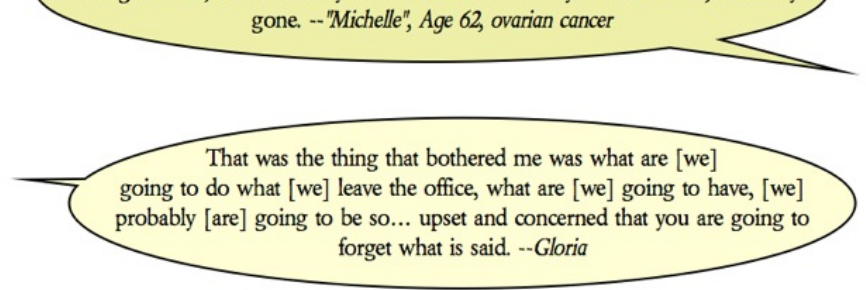

Fig. 2. Sentiments from patients with ovarian cancer, about their first meeting with their oncologist. These patients participated in our design workshops. (Names are aliased).

or visual aids in these discussions. See Fig. 2 for quotes from patients with ovarian cancer we interviewed, expressing sentiment regarding their initial meeting.

2) Identifying in-meeting informational needs: Each workshop incorporated time for each group of participants to identify informational items they felt should be present in the first meetings with oncologists. This included common questions asked to the group by newly diagnosed patients, follow-up questions participants most commonly had for oncologists and their nurse-practitioners, and possibilities for other topic items participants felt would be most important. Flexibility in informational presentation is also a discussion topic for accommodating patient personal preferences into tool design.

3) Design sketching and presentation discussion: Patient participants were asked to actively participate in designing how a shared-decision making tool would function in the oncology clinical setting. In this portion of our workshops, participants provided feedback on physical size, considerations for platforms and physical use of devices, as well as provided considerations for format and layout. Drawings were created on whiteboards collaboratively, with notes in terms of the types of information patients should see in meetings. Some participants also independently created lists of topics or items they thought should be included with varying formats. The contextual use of such interfaces was another discussion topic aimed at determining feasibility and comfort in using such a device in the clinical setting.

4) Take-home informational needs: Participants also provided feedback on the type of take-home information they most wanted when they were first diagnosed. This part of the workshop allowed us to determine key design considerations to prevent overburdening patients with information, while still providing accessibility. Participants also provided concerns for the format and presentation of take-home information, as well as important inclusions for future patients. 


\section{KEY FINDINGS}

Based on the data from our content analysis, we calculated inter-rater reliability using Krippendorf's alpha, which is considered more conservative than other reliability measures, such as Cohen's kappa [34]. For the sessions with oncologists, alpha was .7042, which is considered acceptable according to Hayes and Krippendorf [34]. For patient workshops, alpha was .6580. While this is slightly lower than the recommended value of .667 , we deem it close enough for our design guidelines. (This is an acceptable decision based on Hayes and Krippendorf).

We present the major oncologist and patient concerns below in order of recurrence.

\section{A. Oncologist design considerations}

Our interviews and contextual inquiries with oncologists presented a number of unique design considerations from the oncologist point-of-view. The primary concern oncologists had was meeting patient information needs and promoting their understanding. The next most frequently discussed topics included provisions relating to the design and use of tools for the examination room, communication gaps, and takeaways. These were followed by resources for patients and data maintenance concerns due to the constant change in treatment and cancer-related findings.

1) Meeting information needs: Oncologist participantss believed that text descriptions in decision-making tools should be in layman or non-medical terms. One of our participants suggested the possibility for inexperienced oncologists to give incorrect impressions, which may or may not have a negative effect on patient confidence. This aligns with work discussing the effect of oncologist empathy and attentiveness on patient self-efficacy and confidence [5]. Furthermore, one oncologist expressed interest in addressing patients' needs prior to meetings by providing them tools in the waiting room so that patients may have help preparing questions. The oncologist also thought the possibility of offering audio recording options may be useful for a patient's review later.

2) Promoting patient understanding: Similar to the work conducted by Unruh et al. [20] we found the environment of the exam room is not well suited as an informational workspace for patients. The technological applications of the facility might also need to be considered when designing PCHCTs. Oncologists at the facility we spoke to do not currently have the technological means to digitally store and show patient X-rays or imagery aside from posters; however, they felt that showing imagery is an essential part of promoting patient understanding.

3) Data maintenance concerns: One of the main concerns oncologist participants had in the development of PCHCTs in the clinical setting was the maintenance of data. Information relating to any type of cancer (including the treatment itself) is always in a constant state of flux. Thus, information needs to be current, evidence-based, up-to-date, and automatic from the physician point of view, and yet still be readable in layman terms from the patient's perspective.

4) Resources and takeaways: Oncologist participants were also concerned about the resources and take away information provided to patients. Pamphlets are typically generic, and thus are impersonal to the staging of the patient. Participants suggested providing more personal take-home information based on the patient's diagnosis, staging, and effective treatment. They also suggested providing patients a list of reliable resources either in the area or on the internet.

5) Timing constraints: At the facility where we conducted our interviews, newly diagnosed patient meetings take roughly 30-45 minutes. They scheduled to provide additional time between concurrent meetings and follow-ups. In their current work practice, the secretary may help prepare the file for incoming patients. Thus, the oncologist participants suggested they might need to prepare the communication tool for an oncologist prior to a meeting.

While these work practices may vary based on location, this means the design of shared-decision making and informational tools in the exam room need to be intuitive to prepare prior to patient meetings, as oncologists' time between discussions might be highly variable and may depend on a secretary or nurse to input information.

\section{B. Patient design considerations}

Our design workshops with patients gave the patientperspective in the approach to many of these discussions. The most recurrent themes among our workshops were the inclusion of resources and support, followed by personalization of diagnosis information, information constraints based on personal preferences, design considerations for PCHCTs and their usage, topics of treatment, and physician demeanor.

1) Resources and support: Participants felt the inclusion local resources and support would be beneficial in these meetings. This would enable patients to discuss with doctors both local resources for additional information, local support groups, as well as reputable online resources for information regarding their illness. This local resource inclusion also helps alleviate issues of patients seeking information from less reputable sources. In the case with our participants, the support group provides "bags" to oncologists for those diagnosed with any one of the five types of gynecological cancer to learn more about the group along with informational materials on their illness. Those diagnosed with ovarian cancer prior to the group distributing these bags thought they would have been useful during their own initial meeting, and also reported that of the other types of cancer do not have such resources provided following diagnosis.

Participants also felt that printed copies of discussion topics, along with contacts for support, relevant videos selected by their oncologist, treatment schedules, associated side effects, and terminology definitions be included in their take-home information. Some participants were uninterested in receiving their take-home information in digital form, and thought that such a decision should be made by the patient at the time of their treatment. In the case such a decision is made, participants considered that all information should be made accessible to the patient (such as in the form of an e-mail or document), however, in a collapsed format so that patients do not feel overburdened with information they are not interested in viewing. 
2) Information constraints based on personal preference: In discussing past experiences in the exam room, participants gave insight over discussions where they felt overburdened with information. One patient diagnosed six years ago was still unaware of the meaning of certain terms commonly used by their oncologist, and found it difficult in the beginning to understand all of the information presented to them at once. Some participants express their desire to learn as much as possible following the meeting, while many conveyed disinterest in learning more at the time. Based on this theme, the presentation of information during a clinical visit must be flexible and dynamic, as to allow oncologists to tailor information descriptions on-the-fly. Participants believed accessibility of information during the visit is beneficial to have, though suggested providing options for hiding details as needed.

Furthermore, participants across all workshops thought that information tools in the clinical space should be kept short with regard to the information they display. Many participants expressed worries of time constraints in these settings, and that such platforms should be centered on the main discussion topics defining their illness, and to provide details in the takehome information.

3) Design considerations: In discussing topics participants felt would be most useful in meetings, participants felt that the main five key topics (diagnosis, prognosis, metastasis, and treatment options and side effects) were the most important, though want this information in layman terms. Definitions of these concepts, according to our participants, would thus be an extremely helpful component in building shared informational tools.

Participants also expressed difficulty to visualize many of the concepts doctors would refer to during these meetings. Examination rooms sometimes have posters, but cannot have every type of poster for every part of the body. In addition, many types of cancers, such as multiple myeloma, can manifest in different ways throughout the body. Participants thought using 2D and 3D imagery would be a beneficial component to include in meetings. Visual objects that allowed for manipulation and annotation by oncologists would help users better visualize the location of affected areas and targets for treatment.

The design sketching portions of our workshops created collaborative low-tech prototypes of shared-decision making tools using drawings and whiteboards. Participants offered considerations for the visual representation and use of such a device. Working collaboratively, participants developed a lowtech model for how such an interactive tool might be presented in the clinical environment, as shown in Figure 3.

In addition, participants thought larger interactive displays would be the most useful for an older demographic, however felt such an implementation would be infeasible in current cancer meeting and exam rooms. While computers are present in the exam room, in most cases the computers are geared for the oncologist use for retrieving information, not for patient engagement. Participants thought that if PCHCTs are implemented in the clinical setting, they should be created separate from current systems, and allow for more patient engagement.

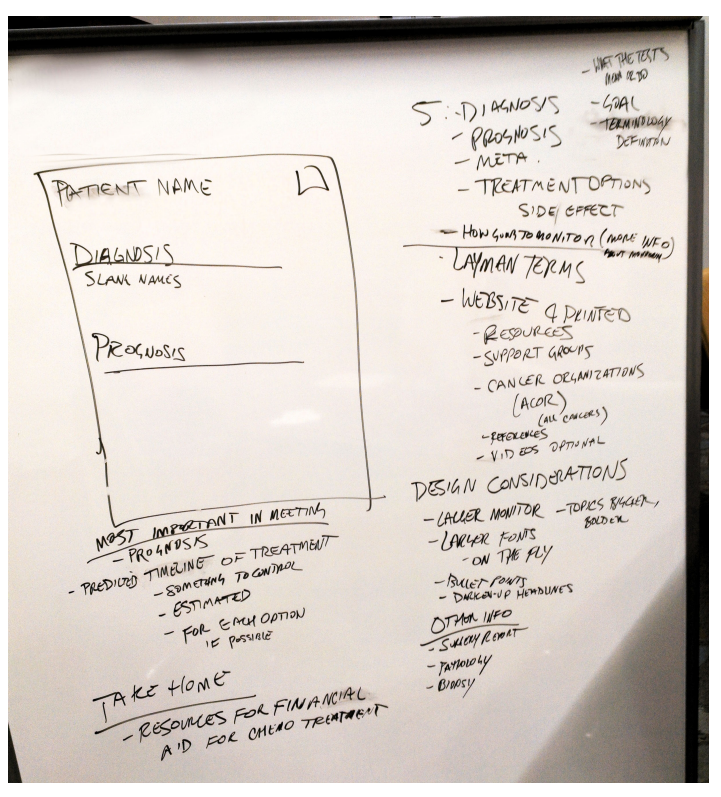

Fig. 3. Sketches from one of the design workshops with patients.

4) Topics of treatment: Patient participants expressed interest in seeing items relating to their treatment schedules in our workshops, as well as items relating to their medications and chemotherapy. Participants also felt that the ability to see an end to their treatments in a visual form would give motivation to continuing it. They also thought the inclusion of key words relating to their specific treatment in layman's terms would help those newly diagnosed to better understand their treatment options.

5) Physician demeanor: Our discussions of past experiences also brought to light oncologist empathetic expression. Some participants discussed past experiences with family members who had undergone cancer treatment with oncologists inexperienced in communicating effectively. Participants suggested notes for oncologists that relay the message they are working with people in an emotionally sensitive setting. Reminders or a communication checklist provided during the preparation stage could be leveraged to remind clinicians to take care to remember the emotional state of the individual, improving patient self-confidence and self-efficacy [3], [4].

\section{DESIGN IMPLICATIONS}

Both patients and provider participants had different opinions for the use of PCHCTs in the exam room. As mentioned previously, one of our oncologist participants suggesting the use of tablet-like tools in the waiting room to help prepare patients for their discussion. The provider suggested patients use these tools to help understand terminology, begin to learn about their illness, and to help them prepare questions prior to their meeting. Patient participants viewed this as unsatisfactory, as the content of these tools might need to be constrained depending on the individual. Furthermore, patient participants also thought that reading this information prior to having their discussions with their doctors could lead to information misinterpretation.

Another suggestion from one of our oncologist participants was to use videos to support patient understanding after the 


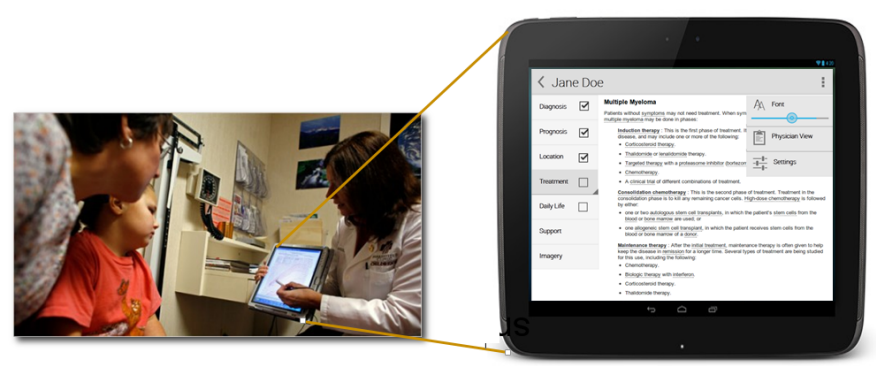

Fig. 4. The first prototype of a patient-centered, shared-decision making health IT tool based on our design workshops with oncologists and patients.

first meeting or prior to follow-ups. While this did not deal directly with applications for PCHCT design, the oncologist thought it could be helpful to provide updateable videos for personalized patient profiles. The oncologist suggested the development of a method for doctors to select among a group of options in a form that will preassign a group of videos for an individual to watch. Patient participants thought this could be problematic if they were not able to ask questions to their healthcare provider, if the video discussed details unrelated to the invididual's condition, or if the video went into more detail than a patient preferred.

We also found conflicts in recommendations with regard to the platform for these tools. The oncologist participants we worked with had an inclination toward tablet and mobile based platforms, while patients thought larger interactive displays would be much more user friendly and visible to older demographics. We suggest that in the development of supplemental interfaces, designers should consider how the tool meets the room, and design with adaptability in mind such that tools can be used in various settings that can accommodate the patient, provider, and environmental constraints. In the case of cancer patients, many of these discussions happen in the exam room, which limits physical space. Adaptable platforms such as Android or Linux systems can enable these tools to be translational among different environments and used on mobile devices, larger interactive displays, and projectable "smartboard" interfaces.

Patient and provider participants both suggested that health information tools can be improved when personalized to the patient. Thus, rather than receiving generic ovarian cancer information, a patient can receive information specific to their diagnosis, prognosis, and treatment plan along with imagery to support their understanding. Furthermore, the interface itself must be adaptable in an on-the-fly manner to accommodate various demographics and preferences. This includes font and image scaling to accommodate various demographics, as well as constraint of information depending on patient preference and tolerance. As many of these discussions can be very emotional to patients, the ability for an oncologist to restrict information is important, so that a patient is not overburdened. In addition, we suggest that designers take care to consider layman terminology in data sources in the development of patientcentered health communication or decision-making tools, or include functionality such that language can be adaptable to the audience (such as clinicians and patients). This aligns with recommendations made by the National Cancer Institute [35].
Due to potential timing constraints of oncologists, accommodations must also be made in the design of PCHCTs and shared decision-making tools for preparation. Oncologists in our study discussed limited time frames between meetings. Intuitive interfaces that leverage pre-populated lists and auto-populate subsections for standardized forms of treatment should be used. Such devices may also require automated methods for updating information, especially since updates to cancer literature can occur within weeks of each other. Designers in this domain should take care in choosing their sources of data to ensure its relevance to the current treatment forms and literature.

The mock-up PCHCT based upon the design considerations we presented is shown in Figure 4, along with an example of a use case in a clinical discussion setting. These interfaces can be useful aides in helping the oncologist better convey concepts relating to their illness. While this figure suggests the use of a tablet, we advise designers take into account the contextual use when designing shared decision-making tools and PCHCTs, considering environmental constraints, user preferences, and clinician workflow.

Finally, patient take-home information was a common discussion topic during our interviews and workshops. Oncologist participants expressed interest in providing digital take-home information for patients that included resources and links to reputable websites when patients do information seeking. Patient participants, however, felt hard copies offered those less familiar with computer-use to have access to their information for reference following their meeting, with digital offerings available based on user-preference. In either case, decisionmaking tools can be tailored to automatically generate these personalized take homes based on in-meeting information to serve as extended material for patients when under less emotional burden. Furthermore, we suggest designers develop their back-end frameworks to be location-aware, to provide patient stakeholders with a means to find local sources for support beyond the clinical setting.

\section{CONCLUSION AND FUture WORK}

In this paper, we presented design considerations for the development of shared decision making and PCHCTs in the cancer discussion setting. Our findings make two primary contributions. First, we utilize PD techniques involving both patient and oncologist stakeholders in the co-design of personalized information tools for newly diagnosed cancer patients. We then used content analysis to determine areas of agreement on the most important topics for stakeholders of these settings to determine design recommendations for shared health informational tool development. Second, we provide considerations in the design of supplementary take-home information for patients based on this in-meeting information.

As more work is done in creating patient-centric software for the clinical setting, the inclusion of both patient and clinician stake-holders in the design process is becoming increasingly essential. While patient needs will always be at the fore-front in the design of tools to support their personal information needs, clinician concerns and recommendations are becoming a core component of design as more applications push into the clinical setting. We plan to continue this work in 
building shared decision-making tools based on our findings, including the development of such a tool that incorporates many of these considerations into its design.

\section{ACKNOWLEDGEMENTS}

We would like to thank our local cancer support group participants, Michiana Hematology and Oncology, Elise Eiden, Alexandra Janiw, Chas Jhin, and Leo Hall for their contributions and support.

\section{REFERENCES}

[1] N. C. Inst, "Seer stat fact sheets: All sites," 2012. [Online]. Available: http://seer.cancer.gov/statfacts/html/all.html

[2] S. Eggly, L. Penner, N. Hagiwara, R. Gonzalez, F. Harper, E. Heath, and T. Albrecht, "Patient, companion, and oncologist agreement regarding information discussed during triadic oncology clinical interactions,' Psycho-Oncology, 2012.

[3] M. A. Stewart, "Effective Physician-Patient Communication and Health Outcomes : A Review," Canadian Medical Association Journal, vol 152, no. 9, 1995.

[4] R. Zachariae, C. G. Pedersen, a. B. Jensen, E. Ehrnrooth, P. B. Rossen, and H. von der Maase, "Association of perceived physician communication style with patient satisfaction, distress, cancer-related self-efficacy, and perceived control over the disease." British journal of cancer, vol. 88, no. 5, Mar. 2003.

[5] T. F. Hack, L. F. Degner, and P. a. Parker, "The communication goals and needs of cancer patients: a review." Psycho-oncology, vol. 14, no. 10, Oct. 2005.

[6] M. Friedrichsen, P. Strang, and M. Carlsson, "Breaking bad news in the transition from curative to palliative cancer carepatient's view of the doctor giving the information," Supportive care in cancer, 2000.

[7] V. Strecher and M. Kreuter, "The effects of computer-tailored smoking cessation messages in family practice settings," ... of Family Practice, no. study 1, 1994.

[8] V. Jenkins, L. Fallowfield, and J. Saul, "Information needs of patients with cancer: results from a large study in UK cancer centres." British journal of cancer, vol. 84, no. 1, Jan. 2001

[9] C. Schaefbauer and K. Siek, "Cautious, but Optimistic: An Ethnographic Study on Location and Content of Primary Care Providers using Electronic Medical Records," Proceedings of the 5th International ICST Conference on Pervasive Computing Technologies for Healthcare, 2011.

[10] G.-M. Breen, T. T. H. Wan, N. J. Zhang, S. S. Marathe, B. K. Seblega, and S. C. Paek, "Improving DoctorPatient Communication: Examining Innovative Modalities Vis-à-vis Effective Patient-Centric Care Management Technology," Journal of Medical Systems, vol. 33, no. 2, Jun. 2008

[11] Y. Chen, V. Ngo, S. Harrison, and V. Duong, "Unpacking exam-room computing: negotiating computer-use in patient-physician interactions," in Proceedings of the 2011 annual conference on Human factors in computing systems. ACM, 2011.

[12] O. Alsos and D. Svanæ s, "Designing for the secondary user experience," Human-Computer Interaction. INTERACT 2011, 2011.

[13] T. Ni and A. Karlson, "AnatOnMe: facilitating doctor-patient communication using a projection-based handheld device," Proceedings of the 2011 Annual Conference on Human Factors in Computing Systems, 2011.

[14] A. M. Piper, R. Campbell, and J. D. Hollan, "Exploring the accessibility and appeal of surface computing for older adult health care support," in Proceedings of the 28th International Conference on Human Factors in Computing Systems - CHI '10. New York, New York, USA: ACM Press, 2010.

[15] Y. Tian and J. D. Robinson, "Incidental health information use and media complementarity: a comparison of senior and non-senior cancer patients." Patient education and counseling, vol. 71, no. 3, Jun. 2008.

[16] P. Klasnja, A. Hartzler, C. Powell, and W. Pratt, "Supporting cancer patients unanchored health information management with mobile technology," vol. 2011, 2011.
[17] P. Klasnja, A. Civan Hartzler, K. T. Unruh, and W. Pratt, "Blowing in the wind: unanchored patient information work during cancer care," in Proceedings of the 28th international conference on Human factors in computing systems. ACM, 2010.

[18] P. Klasnja, A. Hartzler, C. Powell, G. Phan, and W. Pratt, "Health weaver mobile: Designing a mobile tool for managing personal health information during cancer care," vol. 2010, 2010.

[19] L. Littlechild S., Barr, "Using the internet for breast cancer information," in Proceedings of the 2012 International Conference on Communication Healthcare, ser. EACH '12, 2012.

[20] K. T. Unruh, M. Skeels, A. Civan-Hartzler, and W. Pratt, "Transforming clinic environments into information workspaces for patients," in Proceedings of the 28th international conference on Human factors in computing systems. ACM, 2010.

[21] K. T. Unruh and W. Pratt, "The Invisible Work of Being a Patient and Implications for Health Care: "[the doctor is] my business partner in the most important business in my life, staying alive", Conference proceedings. Ethnographic Praxis in Industry Conference, vol. 2008, no. 1, Nov. 2008.

[22] K. Vaajakallio and T. Mattelmäki, "Collaborative design exploration: envisioning future practices with make tools," in Proceedings of the 2007 conference on Designing pleasurable products and interfaces, ser. DPPI '07. New York, NY, USA: ACM, 2007.

[23] J. Vines, M. Blythe, P. Dunphy, V. Vlachokyriakos, I. Teece, A. Monk, and P. Olivier, "Cheque mates: participatory design of digital payments with eighty somethings," in Proceedings of the 2012 ACM Annual Conference on Human Factors in Computing Systems, ser. CHI '12. New York, NY, USA: ACM, 2012.

[24] J. Vines, M. Blythe, S. Lindsay, P. Dunphy, A. Monk, and P. Olivier, "Questionable concepts: critique as resource for designing with eighty somethings," in Proceedings of the 2012 ACM Annual Conference on Human Factors in Computing Systems, ser. CHI '12. New York, NY, USA: ACM, 2012.

[25] S. Lindsay and D. Jackson, "Engaging older people using participatory design," in Proceedings of the 2012 ACM annual conference on Human Factors in Computing Systems - CHI '12. New York, New York, USA ACM Press, 2012.

[26] F. Kensing and J. Blomberg, "Participatory design: Issues and concerns," in Computer Supported Cooperative Work (CSCW), no. 1993, 1998.

[27] M. Muller and S. Kuhn, "Participatory design," Communications of the ACM, 1993.

[28] M. M. Skeels, K. T. Unruh, C. Powell, and W. Pratt, "Catalyzing social support for breast cancer patients," in Proceedings of the SIGCHI Conference on Human Factors in Computing Systems, ser. CHI '10. New York, NY, USA: ACM, 2010.

[29] M. Gonzales and L. Riek, "A Shared Interface to Improve OncologistPatient Communication," in Proceedings of the 6th International Conference on Pervasive Computing Technologies for Healthcare. IEEE, 2012.

[30] M.J. Gonzales and L.D. Riek, “"'Designing an Interface to Support Shared Decision Making in Oncology," in Proceedings of the American Medical Informatics Association (AMIA) workshop on Interactive Systems in Healthcare. AMIA, 2012.

[31] J. P. Hourcade, M. Driessnack, and K. E. Huebner, Proceedings of the 2012 ACM annual conference on Human Factors in Computing Systems - $\mathrm{CHI}$ ' 12 .

[32] S. Uzor, L. Baillie, and D. Skelton, "Senior designers: empowering seniors to design enjoyable falls rehabilitation tools," in Proceedings of the 2012 ACM annual conference on Human Factors in Computing Systems. ACM, 2012.

[33] E. Hargittai, "An update on survey measures of web-oriented digital literacy," Social Science Computer Review, vol. 27, no. 1, 2009.

[34] G. Hayes, G. Abowd, J. Davis, M. Blount, M. Ebling, and E. Mynatt, "Opportunities for pervasive computing in chronic cancer care," Pervasive Computing, 2008

[35] R. Epstein and R. J. Street, "Patient-Centered Communication in Cancer Care: Promoting Healing and Reducing Suffering." National Cancer Institute, NIH Publication No. 07-6225. Bethesda, MD, 2007. 